
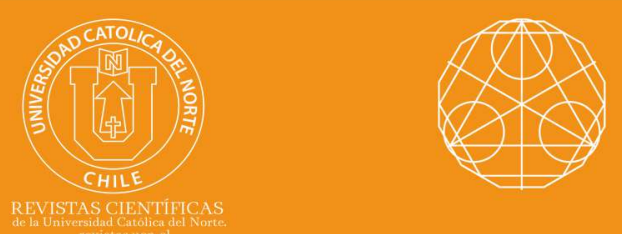

doi"10.22199/issn.0717-6279-2020-06-0098

\title{
On the Mazur-Ulam theorem for Fréchet algebras
}

\author{
A. Zivari-Kazempour ${ }^{1}$ (1) orcid.org/0000-0001-8362-8490 \\ M. R. Omidi ${ }^{2}$ \\ ${ }^{1}$ Ayatollah Borujerdi University, Dept. of Mathematics, Borujerd, Iran. \\ zivari@abru.ac.ir \\ ${ }^{2}$ Kermanshah University of Technology, Dept. of Basic Sciences, Kermanshah, Iran. \\ चm.omidi@kut.ac.ir
}

Received: September 2019 | Accepted: November 2020

\section{Abstract:}

The Mazur-Ulam theorem for Fréchet algebras is proved.

Keywords: Mazur-Ulam Theorem; Fréchet algebras; Isometry; Affine map.

MSC (2020): 46H40, 47A10.

\section{Cite this article as (IEEE citation style):}

A. Zivari-Kazempour and M. R. Omidi, "On the Mazur-Ulam theorem for Fréchet algebras", Proyecciones (Antofagasta, On line),

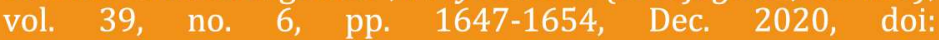

10.22199/issn.0717-6279-2020-06-0098.

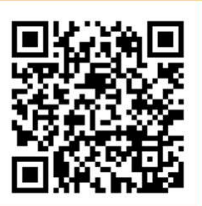

Article copyright: (C) 2020 A. Zivari-Kazempour and M. R. Omidi. This is an open access article distributed under the terms of the Creative Commons License, which permits unrestricted use and distribution provided the original author and source are credited. 


\section{Introduction}

Let $A$ be an algebra over the complex field. A subset $V$ of $A$ is balanced if $\lambda V \subseteq V$ for all scalars $\lambda$ such that $|\lambda| \leq 1$. An algebra $A$ is called a Fré chet algebra if it is a complete metrizable topological linear space and has a neighborhood basis $\left(V_{n}\right)$ of zero such that $V_{n}$ is absolutely convex (convex and balanced) and $V_{n}$ is idempotent, i.e., $V_{n} V_{n} \subseteq V_{n}$ for all $n \in \mathbf{N}$.

The topology of a Fréchet algebra $A$ can be generated by a sequence $\left(p_{n}\right)$ of separating submultiplicative seminorms, i.e.,

$$
p_{n}(x y) \leq p_{n}(x) p_{n}(y)
$$

for all $n \in \mathbf{N}$ and $x, y \in A$, such that $p_{n}(x) \leq p_{n+1}(x)$, whenever $n \in \mathbf{N}$ and $x \in A$.

A Fréchet algebra $A$ with the above generating sequence of seminorms $\left(p_{n}\right)$ is denoted by $\left(A,\left(p_{n}\right)\right)$.

An unital topological algebra $A$ is called a $Q$-algebra if $\operatorname{Inv} A$, the set of all invertible elements of $A$, is open set, or equivalently, Inv $A$ has an interior point in $A$ [6, Lemma E2].

Banach algebras are important examples of Fréchet $Q$-algebras, and Fréchet $Q$-algebras is the well-known class of Fréchet algebras. A Fréchet algebra $A$ is a $Q$-algebra if and only if the spectral radius $r_{A}(x)$ is finite for all $x \in A$. See, for example, [3, Theorem 6.18], [4, III. Proposition 6.2], or [6, Theorem 13.6].

A map $f: E \longrightarrow F$ between real normed spaces is an isometry if for all $x, y \in E,\|f(x)-f(y)\|=\|x-y\|$, and $f$ is affine if for all $x, y \in E$ and $0 \leq t \leq 1$,

$$
f(t x+(1-t) y)=t f(x)+(1-t) f(y) .
$$

An isometry need not be affine. For example, let $\varphi: \mathbf{R} \longrightarrow \mathbf{R}$ be any function such that

$$
|\varphi(s)-\varphi(t)| \leq|s-t|,
$$

for all $s, t \in \mathbf{R}$, such as $\varphi(t)=|t|$. Define $f: \mathbf{R} \longrightarrow \mathbf{R}^{2}$ by $f(x)=(x, \varphi(x))$, where $\mathbf{R}^{2}$ equipped with the norm $\|x\|=\max \left\{\left|x_{1}\right|,\left|x_{2}\right|\right\}$. Then $f$ is an isometry, but it is not affine.

There are two important cases when every isometry is affine:

(i) $\mathrm{F}$ is strictly convex, i.e., if $x=y$ and $\|x\|=\|y\|=1$ together imply that 
$\|x+y\|<2$. Then for all $a, b \in E$,

$$
f\left(\frac{a+b}{2}\right)=\frac{f(a)+f(b)}{2} .
$$

Thus, $f: E \longrightarrow F$ is affine. For example, every inner product space and the spaces $l^{p}$ for $1<p<\infty$, are strictly convex.

(ii) $\mathrm{f}$ is bijective. This famous result was proved by S. Mazur and S. Ulam

[5] in 1932, and their proof is also given in the book [1, p. 166].

In other words, they proved the following well-known Theorem.

Theorem 1.1. Every bijective isometry between real normed spaces $E$ and $F$ is affine.

For a simple proof of this result, see [8] and [7]. Theorem 1.1 was improved by relaxing the subjectivity condition in [2] as follows.

Theorem 1.2. Let $E, F$ be normed spaces and let $f$ be an isometry of $E$ into $F$. Assume that for every unit vector $y \in F$ there exist $a, b \in E$ and $a$ real number $\lambda$ such that

$$
\|y-\lambda(f(a)-f(b))\|<\frac{1}{2}
$$

Then $f$ is affine and $f(E)$ is a dense subspace of $F$. Moreover, if $E$ is complete, then $f(E)=F$.

In this paper, we prove the classical Mazur-Ulam Theorem for Fréchet algebras.

\section{Mazur-Ulam Theorem for Fréchet algebras}

Let $\left(A,\left(p_{n}\right)\right)$ and $\left(B,\left(q_{n}\right)\right)$ be Fréchet algebras. A map $f:\left(A,\left(p_{n}\right)\right) \longrightarrow$ $\left(B,\left(q_{n}\right)\right)$ is called isometry if

$$
p_{n}(x-y)=q_{n}(f(x)-f(y)),
$$

for all $n \in \mathbf{N}$ and every $x, y \in A$, and $f$ is called affine if

$$
f(\lambda x+(1-\lambda) y)=\lambda f(x)+(1-\lambda) f(y),
$$

for all $\lambda \in[0,1]$ and every $x, y \in A$.

Theorem 2.1. Let $X$ and $Y$ be two real vector spaces. Then the following statements are equivalent: 
(i) The map $f: X \longrightarrow Y$ is affine.

(ii) If $\sum_{k=1}^{n} \lambda_{k}=1$, then

$$
f\left(\sum_{k=1}^{n} \lambda_{k} x_{k}\right)=\sum_{k=1}^{n} \lambda_{k} f\left(x_{k}\right)
$$

(iii) The map $T: X \longrightarrow Y$, defined by $T(x)=f(x)-f(0)$ is $\mathbf{R}$-linear.

Proof. $(i) \Longrightarrow(i i)$. It follows from induction.

(ii) $\Longrightarrow($ iii $)$. Let $x \in X$ and $\lambda \in \mathbf{R}$ be arbitrary. Then

$$
\begin{aligned}
T(\lambda x) & =f(\lambda x)-f(0) \\
& =f(\lambda x+(1-\lambda) 0)-f(0) \\
& =\lambda f(x)+(1-\lambda) f(0)-f(0) \\
& =\lambda T(x) .
\end{aligned}
$$

Thus, for all $x \in X$ and $\lambda \in \mathbf{R}$,

$$
T(\lambda x)=\lambda T(x) .
$$

By assumption and (2.1), we have

$\frac{1}{2} T(x+y)=T\left(\frac{x+y}{2}\right)=f\left(\frac{x+y}{2}\right)-f(0)=\frac{1}{2} f(x)+\frac{1}{2} f(y)-f(0)=\frac{1}{2}(T(x)+T(y))$.

Hence $T$ is additive, and so it is $\mathbf{R}$-linear.

$($ iii $) \Longrightarrow(i)$. Assume that $\lambda \in[0,1]$ and $x, y \in X$. Then

$$
\begin{aligned}
f(\lambda x+(1-\lambda) y) & =T(\lambda x+(1-\lambda) y)+f(0) \\
& =\lambda T(x)+(1-\lambda) T(y)+f(0) \\
& =\lambda T(x)+(1-\lambda) T(y)+(\lambda+(1-\lambda)) f(0) \\
& =\lambda f(x)+(1-\lambda) f(y) .
\end{aligned}
$$

Thus, the map $f: X \longrightarrow Y$ is affine.

Lemma 2.2. Let $f: X \longrightarrow Y$ be a continuous map between topological vector spaces. Then $f$ is affine if for all $a, b \in X$, 


$$
f\left(\frac{a+b}{2}\right)=\frac{f(a)+f(b)}{2} .
$$

Proof. By Theorem 2.1, it is enough to prove that the map $T: X \longrightarrow Y$, defined by $T(x)=f(x)-f(0)$ is $\mathbf{R}$-linear. Take $b=0$ in (2.2), gives

$$
f\left(\frac{a}{2}\right)=\frac{f(a)+f(0)}{2} .
$$

Hence

$$
T\left(\frac{x}{2}\right)=f\left(\frac{x}{2}\right)-f(0)=\frac{f(x)+f(0)}{2}-f(0)=\frac{1}{2} T(x) .
$$

Replacing $x$ by $x+y$ in (2.3), we get

$\frac{1}{2} T(x+y)=T\left(\frac{x+y}{2}\right)=f\left(\frac{x+y}{2}\right)-f(0)=\frac{1}{2}(f(x)+f(y))-f(0)=\frac{1}{2}(T(x)+T(y))$.

Thus, for all $x, y \in X$,

$$
T(x+y)=T(x)+T(y),
$$

and so $T$ is additive. Now by a simple calculation it follows from the additivity and continuity of $T$ that it is $\mathbf{R}$-linear and so $f$ is affine.

For $a \in A$, the reflection of $A$ in $a$ is the map $\delta: A \longrightarrow A$ defined by $\delta(x)=2 a-x$. Then $\delta \circ \delta$ is the identity, and hence $\delta$ is bijective with $\delta^{-1}=\delta$. Moreover, $\delta$ is an isometry and $a$ is the only fixpoint of $\delta$. The equations

$$
p_{n}(\delta(x)-a)=p_{n}(x-a), \quad p_{n}(\delta(x)-x)=2 p_{n}(x-a),
$$

hold for all $x \in A$.

Now we state and prove the main theorem.

Theorem 2.3. Let $T:\left(A,\left(p_{n}\right)\right) \longrightarrow\left(B,\left(q_{n}\right)\right)$ be an bijective isometry between Fréchet algebras. Then $T$ is affine.

Proof. Let $a, b \in A$ and set $x=\frac{1}{2}(a+b)$. Let $L$ be the family of all bijective isometries $g: A \longrightarrow A$ sush that $g(a)=a$ and $g(b)=b$. Set

$$
\alpha=\sup \left\{p_{n}(g(x)-x): g \in L\right\} .
$$


For $g \in L$ we have

$$
p_{n}(g(x)-a)=p_{n}(g(x)-g(a))=p_{n}(x-a),
$$

and hence

$$
p_{n}(g(x)-x) \leq p_{n}(g(x)-a)+p_{n}(a-x)=2 p_{n}(a-x),
$$

which prove that $\alpha$ is finite.

Let $\delta$ be the reflection of $A$ in $x$. If $g \in L$, then also $g^{*}=\delta \circ g^{-1} \circ \delta \circ g \in L$, and hence $p_{n}\left(g^{*}(x)-x\right) \leq \alpha$. Since $g^{-1}$ is an isometry, by (2.4), for all $g \in L$ we get

$$
\begin{aligned}
2 p_{n}(g(x)-x) & =p_{n}(\delta \circ g(x)-g(x)) \\
& =p_{n}\left(g^{-1} \circ \delta \circ g(x)-x\right) \\
& =p_{n}\left(\delta \circ g^{-1} \circ \delta \circ g(x)-x\right) \\
& =p_{n}\left(g^{*}(x)-x\right) \leq \alpha,
\end{aligned}
$$

and hence $2 \alpha \leq \alpha$. Thus, $\alpha=0$ and so $p_{n}(g(x)-x)=0$ for all $g \in L$. Since $\left(p_{n}\right)$ separate the point of $A$ we get $g(x)=x$. Let $T: A \longrightarrow B$ be a bijective isometry. Setting

$$
y=\frac{1}{2}(T(a)+T(b)) .
$$

Let $f$ be the reflection of $B$ in $y$. Then the map $h=\delta \circ T^{-1} \circ f \circ T$ is in $L$, and hence $h(x)=x$. This implies that $f \circ T(x)=T(x)$. Since $y$ is the only fix point of $f$, we obtain $T(x)=y$, and $T$ is affine by Lemma 2.2.

\section{Strictly Convex case}

A Fréchet algebra $\left(A,\left(p_{n}\right)\right)$ is called strictly convex if $x, y \in A \backslash\{0\}$ and

$$
p_{n}(x+y)=p_{n}(x)+p_{n}(y),
$$

for all $n \in \mathbf{N}$, imply that $x=\lambda y$ for some $\lambda>0$.

Lemma 3.1. Let $\left(A,\left(p_{n}\right)\right)$ be a strictly convex Fréchet algebra, and $x, y \in$ $A$. Then $u=\frac{1}{2}(x+y)$ is the unique element of $A$ such that for all $n \in \mathbf{N}$,

$$
2 p_{n}(x-u)=2 p_{n}(y-u)=p_{n}(x-y) .
$$


Proof. The result is clear if $x=y$, and it is also easy to see that (3.1) holds. It is suffices to show that $u$ is unique. Suppose that $x=y$ and $u, w \in A$ with

$$
2 p_{n}(x-u)=2 p_{n}(y-u)=p_{n}(x-y)=2 p_{n}(x-w)=2 p_{n}(y-w) .
$$

Then

$$
p_{n}(2 x-(u+w)) \leq p_{n}(x-u)+p_{n}(x-w)=p_{n}(x-y) .
$$

Similarly,

$$
p_{n}(2 y-(u+w)) \leq p_{n}(x-y) .
$$

If either of inequalities (3.2) or (3.3) is strict, then we must have

$$
p_{n}(2 x-2 y) \leq p_{n}(2 x-(u+w))+p_{n}(2 y-(u+w))<2 p_{n}(x-y),
$$

which is contradiction. Thus, equality holds in (3.2) and (3.3). So

$$
p_{n}((x-u)+(x-w))=p_{n}(x-y)=p_{n}((y-u)+(y-w)) .
$$

Since $\left(A,\left(p_{n}\right)\right)$ is strictly convex, $x=u$ and $x=w$, it follows that $(x-u)=\lambda(x-w)$ for some $\lambda>0$. On the other hand, $p_{n}(x-u)=p_{n}(x-w)$, so $\lambda=1$. Therefore $u=w$. This completes the proof.

Now we have the following result.

Theorem 3.2. Every isometry from Fréchet algebra $\left(A,\left(p_{n}\right)\right)$ into strictly convex Fréchet algebra $\left(B,\left(q_{n}\right)\right)$ is affine.

Proof. Let $T:\left(A,\left(p_{n}\right)\right) \longrightarrow\left(B,\left(q_{n}\right)\right)$ be an isometry. Then we have

$$
q_{n}(T(x)-T(y))=p_{n}(x-y)=2 p_{n}\left(\frac{x-y}{2}\right)=2 q_{n}\left(T\left(\frac{x+y}{2}\right)-T(x)\right) .
$$

Similarly,

$$
q_{n}(T(x)-T(y))=p_{n}(x-y)=2 q_{n}\left(T\left(\frac{x+y}{2}\right)-T(y)\right),
$$

for all $x, y \in A$. It follows from Lemma 3.1 that

$$
T\left(\frac{x+y}{2}\right)=\frac{1}{2}(T(x)+T(y)),
$$


for all $x, y \in A$. Thus, $T$ is affine by Lemma 2.2 .

\section{Acknowledgments}

The author would like to thank the referees for proving valuable comments and helping in improving the content of this paper.

\section{References}

[1] S. Banach, Theorie des opérations lineaires. Warsaw, 1932.

[2] T. Figiel, P. Šemrl, and J. Väisälä, "Isometries of normed spaces", Colloquium mathematics, vol. 92, no. 1, pp. 153-154, 2002, doi: $10.4064 / \mathrm{cm} 92-1-13$

[3] M. Fragoulopoulou, Topological algebras with involution. Burlington: North-Holland, 2005.

[4] A. Mallios, Topological algebras, Burlington: North-Holland, 1986.

[5] S. Mazur and S. Ulam, Sur les transformations isométriques d'espaces vectoriels normés, Comptes Rendus de l'Académie des Sciences Paris, vol. 194, pp. 946-948, 1932.

[6] E. A. Michael, Locally multiplicatively convex topological algebras, Providence, RI: American Mathematics Society, 1952.

[7] B. Nica, "The Mazur-Ulam theorem", Expositiones mathematicae, vol. 30, no. 4, pp. 397-398, 2012, doi: 10.1016/ j.exmath.2012.08.010

[8] J. Väisälä, "A proof of the Mazur-Ulam theorem", The American mathematical monthly, vol. 110, no. 7, pp. 633-635, 2003, doi: $10.2307 / 3647749$ 